\title{
Probing the hydrogen-bond network of water via time-resolved soft $x$-ray spectroscopy
}

\author{
Nils Huse, ${ }^{* a}$ Haidan Wen, ${ }^{\sharp b}$ Dennis Nordlund, ${ }^{c}$ Erzsi Szilagyi ${ }^{b d}$ Dan Daranciang, ${ }^{b d}$ Timothy A. Miller, ${ }^{b e}$ \\ Anders Nilsson, ${ }^{c f}$ Robert W. Schoenlein ${ }^{a g}$ and Aaron M. Lindenberg ${ }^{b e}$
}

DOI: $10.1039 / b 822210 j$

We report time-resolved studies of hydrogen bonding in liquid $\mathrm{H}_{2} \mathrm{O}$, in response to direct excitation of the $\mathrm{O}-\mathrm{H}$ stretch mode at $3 \mu \mathrm{m}$, probed via soft $\mathrm{x}$-ray absorption spectroscopy at the oxygen K-edge. This approach employs a newly developed nanofluidic cell for transient soft $\mathrm{X}$-ray spectroscopy in liquid phase. Distinct changes in the near-edge spectral region (XANES) are observed, and are indicative of a transient temperature rise of $10 \mathrm{~K}$ following transient laser excitation and rapid thermalization of vibrational energy. The rapid heating occurs at constant volume and the associated increase in internal pressure, estimated to be $8 \mathrm{MPa}$, is manifest by distinct spectral changes that differ from those induced by temperature alone. We conclude that the near-edge spectral shape of the oxygen $\mathrm{K}$-edge is a sensitive probe of internal pressure, opening new possibilities for testing the validity of water models and providing new insight into the nature of hydrogen bonding in water.

\section{Introduction}

Understanding the structure of water and the associated dynamics is of paramount importance due to the outstanding role of water in living organisms and the biosphere in general. Water structure and dynamics are intimately related to hydrogen bonding which gives rise to a dynamic network that permeates the entire liquid and deviates from the tetrahedral structure of ice $^{1}$. For this reason, water has been investigated by various experimental techniques that probe observables sensitive to hydrogen bonding, among them $\mathrm{x}$-ray and vibrational spectroscopy ${ }^{2,3}$. Although these techniques probe very different energy scales, they share chemical specificity and local character. An important distinction is that x-ray spectroscopy probes local structure via core level transitions with atomic sensitivity and element specificity, whereas infrared spectroscopy, also being chemically specific, is local on length scales of vibrational modes in molecules. Static $\mathrm{x}$-ray spectroscopy has revealed quantitative information on local atomic structure ${ }^{4,5}$ whereas time-resolved vibrational spectroscopy has produced information on timescales of structural dynamics as short as $50 \mathrm{fs}$. $6,7,8,9$

Time-resolved vibrational spectroscopy has concentrated on the infrared spectrum of HOD in $\mathrm{D}_{2} \mathrm{O}$ and $\mathrm{H}_{2} \mathrm{O}^{10,11,12,13,14,15,16}$ and to a lesser extent on pure $\mathrm{H}_{2} \mathrm{O},{ }^{17,18}$ limited by the (sub-)micron absorption length. The latter also poses a challenge for x-ray absorption spectroscopy. ${ }^{2}$ The infrared spectrum of a $400 \mathrm{~nm}$ thick water sample is shown in Fig. 1A. ${ }^{19}$ Studies to date have focused on the $\mathrm{OH}$ stretching vibration centred at $\mathrm{v}_{\mathrm{OH}}=3400 \mathrm{~cm}^{-}$ ${ }^{1}$ because of its sensitivity to hydrogen bonding. More recently, the $\mathrm{OH}$ bending vibration $\delta_{\mathrm{OH}}$ in $\mathrm{H}_{2} \mathrm{O}$ and $\mathrm{D}_{2} \mathrm{O}$ and librational modes $v_{\mathrm{L}}$ between $650 \mathrm{~cm}^{-1}$ and $1800 \mathrm{~cm}^{-1}$ have been investigated by femtosecond vibrational spectroscopy. ${ }^{20,21,22,23,24}$ There is also abundant literature on the low-frequency spectral response of water between a few wavenumbers and the $\mathrm{O} \cdots \mathrm{O}$ stretching vibration $v_{\mathrm{O} \ldots \mathrm{O}}{ }^{25,26,27,28,29,30}$

Static x-ray spectroscopy experiments have been used to investigate the oxygen $\mathrm{K}$-edge of water, the near-edge region of which is shown in Fig. 1B. Of particular significance are the pre-edge (I), main-edge (II) and post-edge (III) regions due to their distinct sensitivity to the local atomic structure and hydrogen bonding. ${ }^{4,5}$ Various techniques have been employed to measure this spectral region such as x-ray Raman scattering (XRS) and X-ray absorption spectroscopy (XAS) using transmission, fluorescence and total and Auger electron yield (TEY/AEY). ${ }^{31,32,33,34,35}$

Interpretations of $\mathrm{x}$-ray spectra of liquid water have been based on direct comparison of the absorption in the near-edge region of the oxygen K-edge with that of bulk-ice, surface layers of ice, and gas-phase water. More detailed interpretations of water XAS in conjunction with model calculations of the local hydrogen-bonding structure have led to much discussion about the validity and ambiguity of various water models. ${ }^{36,37,38,39,40,41,42,43}$ Generally, quantitative interpretation of spectroscopic data often requires theoretical modelling. This is particularly true when structural information of liquid water is extracted from spectral data. In this respect, vibrational spectroscopy has been compromised by ambiguous correspondence between frequency and structure; notwithstanding new multidimensional techniques that separate overlapping spectral contributions along several dimensions in frequency space. ${ }^{6,7,8}$

Recently, x-ray diffraction in liquids has been implemented in a time-resolved manner to investigate structural dynamics and chemical processes in the liquid-phase. ${ }^{44,45,46} \mathrm{X}$-ray diffraction provides the Fourier transform of the electron density which, in the case of water corresponds primarily to the $\mathrm{O}-\mathrm{O}$ radial distribution function. However, the limited available Q-range has often precluded direct inversion of diffraction data and interpretation has also relied on model simulations. The main limitation of diffraction techniques is the lack of chemical specificity, restricting liquid-phase experiments to systems with acceptable scattering contrast between solute and solvent or the study of the latter itself.

We seek new information on water structure by using transient XAS, which can distinguish between sub-ensembles of water molecules by selective excitations, to link local water 

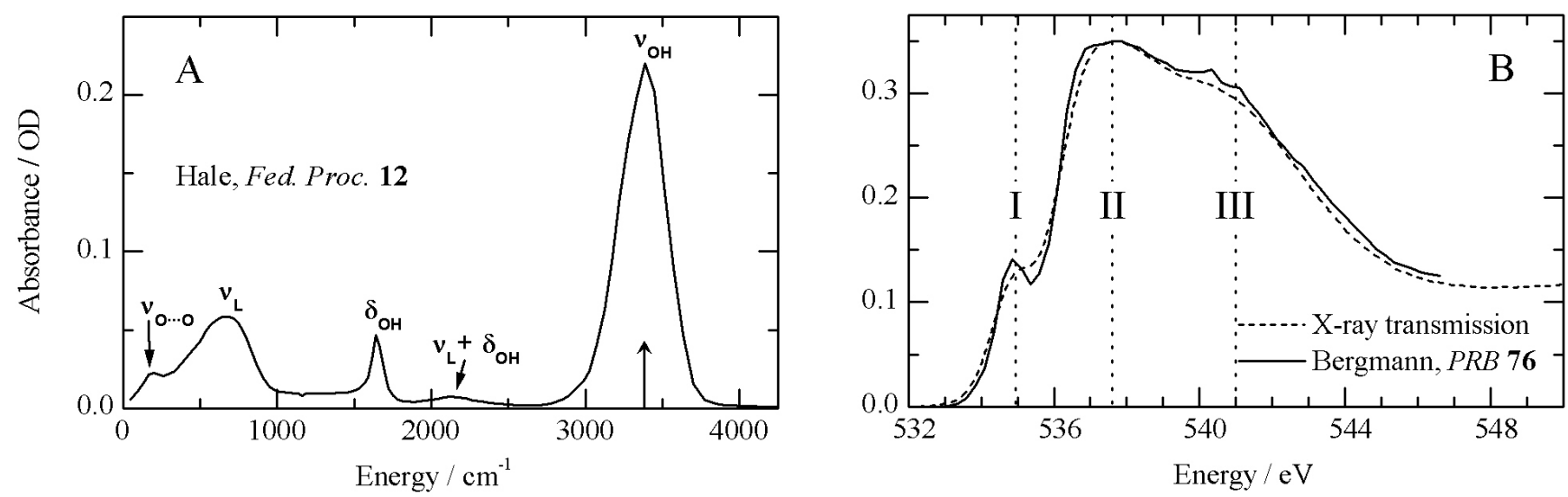

Fig. 1 A. Static infrared absorption spectrum of a $400 \mathrm{~nm}$ thick water film between $50 \mathrm{~cm}^{-1}$ and $4250 \mathrm{~cm}^{-1}$ (from reference 19 ). The vertical arrow at $3400 \mathrm{~cm}^{-1}$ indicates the spectral position of the infrared pump pulse. B. Static x-ray absorption spectrum of water in the near-edge region of the oxygen K-edge measured in transmission (400 $\mathrm{nm}$ thick film, solid curve) and by Raman scattering (dashed curve from reference 50 , rescaled) at $22{ }^{\circ} \mathrm{C}$. The vertical lines mark absorption maxima and the corresponding regions around them are commonly referred to as the pre-edge (I), main-edge (II), and post-edge (III) of the water Xray spectrum. The post-edge region connects to the extended $\mathrm{x}$-ray absorption fine structure (EXAFS).

structures to X-ray absorption spectra. While future femtosecond soft $\mathrm{x}$-ray XAS will allow to monitor water molecules in nonequilibrium conditions such as vibrationally excited water, picosecond transient XAS is very sensitive to changes in macroscopic variables such as temperature and pressure allowing for previously inaccessible experimental sensitivity.

We have developed a solution-phase infrared pump/soft x-ray probe experiment at the Advanced Light Source (ALS). The short absorption lengths in the soft $x$-ray region have complicated time-resolved XAS in the liquid phase because of the significant experimental challenge to create sub-micron liquid films that remain stable in a vacuum environment under typical photo-excitation conditions. We have designed a nanofluidic cell for soft $x$-ray spectroscopy in liquid phase that allows for sub-200nm control of the liquid film thickness anywhere between $200 \mathrm{~nm}$ and tens of microns. ${ }^{18,47}$

In this paper, we present time-resolved XAS studies of vibrationally excited water, and compare them to temperaturedependent $\mathrm{x}$-ray spectra of water obtained via Raman scattering. Measurements over time scales from 150 ps to $200 \mathrm{~ns}$ reveal the evolution of hydrogen bonding in response to transient changes in both temperature and pressure.

\section{Experimental}

Transient $\mathrm{x}$-ray absorption measurements were performed at the soft $x$-ray femtosecond beamline 6.0.2 at the ALS. ${ }^{48,49} \mathrm{~A}$ schematic of the beamline is shown in Fig. 2. It consists of two parts, a high-repetition-rate $800 \mathrm{~nm}$ Ti:sapphire laser system that produces frequency-converted infrared pulses and an undulatorbased soft x-ray beamline, equipped with an x-ray chopper and a varied line space grating monochromator, tuneable from $200 \mathrm{eV}$ to $2 \mathrm{keV}$.

The filling pattern of the ALS synchrotron consists of 276 electron bunches spaced $2 \mathrm{~ns}$ apart with one "camshaft" bunch (10mA max. current, 70ps full width half maximum bunch duration) isolated in a 100ns gap to allow for gated detection of the $\mathrm{x}$-rays produced by this single bunch. Differential $\mathrm{x}$-ray absorption spectra at the oxygen K-edge were recorded by tuning the energy between $530 \mathrm{eV}$ and $550 \mathrm{eV}$ using a monochromator resolution of $0.5 \mathrm{eV}$. The $\mathrm{x}$-ray pulses are focused to a spot size of $50 \mu \mathrm{m}$ with a flux of about $10^{4}$ photons/pulse/ $0.1 \%$ bandwidth at the sample. The X-rays were attenuated by a factor of 10 to ensure stable sample conditions.

The $150 \mathrm{fs}$ pump pulses at $2.95 \mu \mathrm{m}$ wavelength are derived from an optical parametric amplifier (Light Conversion TOPAS$\mathrm{C}$ ), and are synchronized to the x-ray pulses by locking the $62.5 \mathrm{MHz}$ repetition rate of the cavity-length-stabilized oscillator of the Ti:sapphire laser system to the $500 \mathrm{MHz}$ repetition rate of the radio frequency cavity of the synchrotron, with a precision of better than a few picoseconds. The spectrum of the pump pulses is determined by measuring the frequency-doubled signal of the infrared pulses with a grating spectrometer (Ocean Optics NIR256). The infrared pump pulses are focused to $150 \mu \mathrm{m}$ at the sample $\left(4 \mu \mathrm{J} /\right.$ pulse, incident at $\sim 10^{\circ}$ with respect to the $\mathrm{x}$-rays)
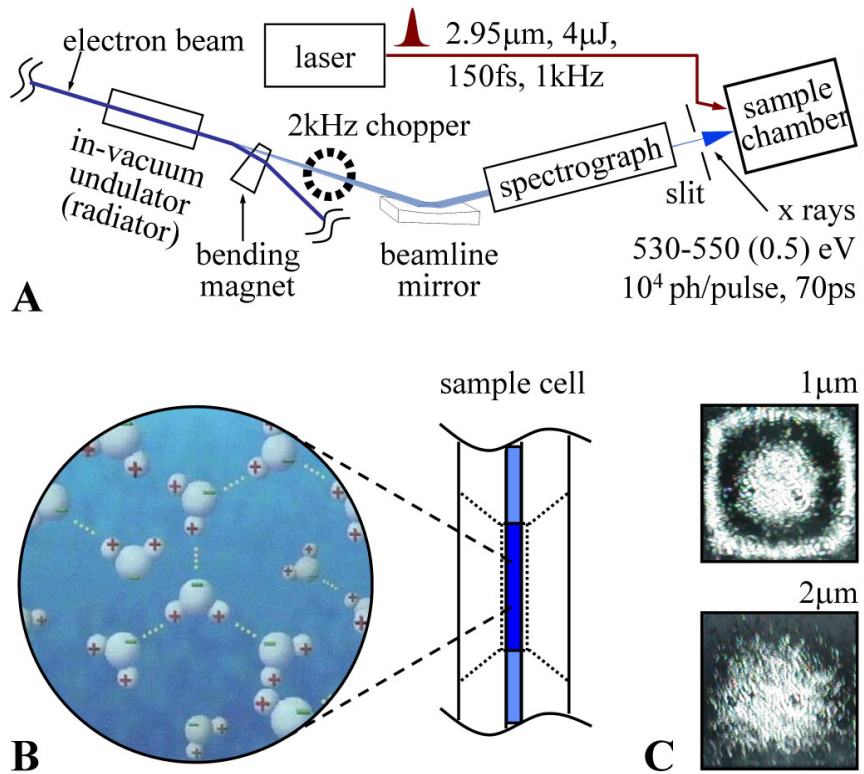

Fig. 2 A. Schematic of ALS beamline 6.0.2. B. Cross section of the central part of the sample cell in which a liquid water film is sandwiched between two silicon nitride membranes. The pressure inside the sample volume is controlled while the chamber is backfilled with helium to constant pressure. C. Two transmission interferograms are shown which serve as a measure of the liquid film thickness with sub-200nm accuracy. 
and are detected outside the vacuum chamber with a PbSe diode for alignment purposes.

Spatial overlap of infrared pump and x-ray probe pulses is ensured by alignment through a pinhole placed in the sample plane. Temporal overlap with $<100$ ps accuracy is determined byusing a fast silicon avalanche photodiode and a $\mathrm{GHz}$ oscilloscope (Tektronix 5104B). For delay scans at fixed x-ray energy, the time delay between pump and probe pulses is varied electronically with picosecond accuracy. The $\mathrm{OH}$ stretching vibration is excited by the infrared pulses into its first excited state $v_{\mathrm{OH}}=1$ at a repetition rate of $1 \mathrm{kHz}$ while the $\mathrm{x}$-ray pulses that probe the sample transmission were recorded at $2 \mathrm{kHz}$ thereby creating interleaved data of unperturbed and the excited sample transmission, I and $I(t)$, respectively, $t$ being the time delay between the pump and the probe pulses. The total integration time per data point is typically $20 \mathrm{~s}$.

The liquid film sample is held in a $0.5 \mathrm{~mm}$-wide channel between two $100 \mathrm{~nm}$-thick silicon nitride membranes as pictured in Fig. 2. The entire sample cell is mounted in a vacuum chamber that is filled with moderate helium pressure to offset the vapour pressure of the liquid. The sample chamber is isolated from the beamline vacuum by a third 100nm-thick silicon nitride membrane. The thickness of the liquid film is optimized by controlling the sample cell pressure, which is typically set to 150 torr.

Although it is difficult to determine the exact equilibrium temperature of the sample under continuous infrared pumping, consideration of the boiling point provides an upper limit. Even at 100torr, where the boiling point of water is $50^{\circ} \mathrm{C}$ as inferred from the Clausius-Clapeyron equation, we did not observe any boiling of the sample. We therefore conclude that our equilibrium sample temperature is between room temperature and $50^{\circ} \mathrm{C}$. The typical sample thickness for these experiments is $400 \mathrm{~nm}$, which is slightly less than the absorption length of the $\mathrm{x}$-ray pulses at $537.5 \mathrm{eV}$. The infrared absorption is well matched to that of the X-rays, amounting to an optical density of about 0.2 (see Fig. 1 A and B absorption of infrared light and X-rays). We also tested the behaviour of thinner and thicker samples.

An x-ray Raman spectrum of water at $22{ }^{\circ} \mathrm{C}$ at the oxygen $\mathrm{K}$ edge $^{50}$ is shown for comparison in Fig. 1B (dashed line). The static absorption spectrum in Fig. 1B (solid line) is in good agreement. Differences between the two spectra in the pre-edge

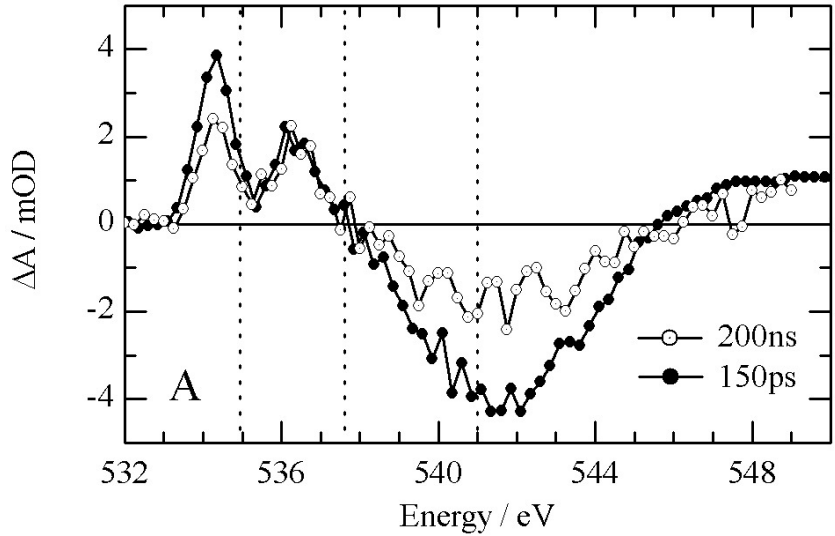

region are due to the coarser energy resolution of $1 \mathrm{eV}$ in the $\mathrm{x}$ ray Raman scattering experiment as compared to the $0.5 \mathrm{eV}$ resolution in this work. Slight fluctuations in sample thickness in the XAS experiment account for differences in the main- and post-edge region. Generally, the two techniques yield identical results although XAS measures the attenuation of X-rays that pass through the sample as a function of incident $\mathrm{X}$-ray energy while XRS measures the intensity of X-rays with energies far above the absorption edge that are inelastically scattered in the sample as a function of energy shift between the incident and the scattered X-rays.

The absorbance A of the unexcited sample is calculated from the incident and the transmitted X-rays, $\mathrm{I}_{0}$ and $\mathrm{I}$, according to $\mathrm{A}$ $=-\log \left(\mathrm{I} / \mathrm{I}_{0}\right)$. The change in absorbance $\Delta \mathrm{A}$ is calculated from the transmitted $\mathrm{x}$-ray intensities of the unperturbed and the excited sample with $\Delta \mathrm{A}=-\log (\mathrm{I}(\mathrm{t}) / \mathrm{I})$. In the small signal limit, $\Delta \mathrm{A}=$ $(\ln 10)^{-1}(\Delta \mathrm{I} / \mathrm{I})$ where $\Delta \mathrm{I}=\mathrm{I}(\mathrm{t})-\mathrm{I}$. Then, a relative transmission change $\Delta \mathrm{T} / \mathrm{T}=\Delta \mathrm{I} / \mathrm{I}=1.0 \%$ corresponds to an absorbance change $\Delta \mathrm{A}=4.3 \mathrm{mOD}$.

\section{Results and discussion}

We recorded time-resolved $\mathrm{x}$-ray absorption data between $532 \mathrm{eV}$ and $550 \mathrm{eV}$ covering the pre- (I), main- (II), and postedge region (III) of the oxygen $\mathrm{K}$-edge. The results are plotted as differential absorption spectra at delays of $150 \mathrm{ps}$ and $200 \mathrm{~ns}$ in Fig. 3A (solid and open circles). At both delays, pronounced increases in absorption $(\Delta \mathrm{A}>0)$ are apparent in the pre- and main-edge region with maximal changes at $534.4 \mathrm{eV}$ and $536.4 \mathrm{eV}$. It should be noted that these maxima occur at the rising edge of the pre- and main-edge peaks of the K-edge spectrum, indicating enhanced peak absorption and a shift of the pre- and main edge absorption maxima towards lower energies. A broad absorption decrease $(\Delta \mathrm{A}<0)$ in the post-edge region between $538 \mathrm{eV}$ and $545 \mathrm{eV}$ is followed at higher energies by an absorption increase beyond $545 \mathrm{eV}$. This absorption increase is flat beyond $548 \mathrm{eV}$ and extends to at least $556 \mathrm{eV}$ (measured at $150 \mathrm{ps}$, data not shown). Clearly, absorption is shifted away from the centre of the post-edge region at $542 \mathrm{eV}$ towards the pre- and main-edge region and to energies beyond $545 \mathrm{eV}$. The differential absorption spectrum continues to evolve for pumpprobe delays $\mathrm{t}>150 \mathrm{ps}$, as evidenced by spectral measurements at

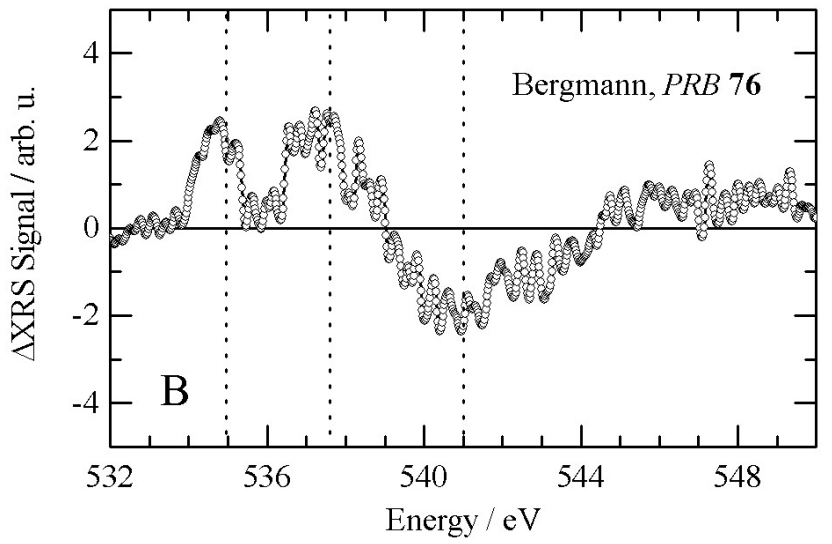

Fig. 3 Transient absorption change of the oxygen K-edge after excitation of the $\mathrm{OH}$ stretching vibration of water. A. Differential absorption spectrum at two infrared pump/x-ray probe delays of 150ps (solid circles) and 200ns (open circles). B. Difference of two static Raman spectra recorded at temperatures of $2^{\circ} \mathrm{C}$ and $22^{\circ} \mathrm{C}$ taken from reference 44 , rescaled by 1.2 , and reversed in sign. 
200ns delay. Surprisingly, the dynamics on timescales $>150 \mathrm{ps}$ are only observed in the pre- and post-edge region whereas the absorbance change in the main-edge region remains constant between 150ps and 200ns. The origin of the long-time dynamics is discussed below.

We note that during the course of this experiment Wernet et al. ${ }^{51}$ published data consistent with the differential absorption spectrum at 150 ps delay in Fig 3A. However, the limited timerange of the former experiments prevented the clear observation of the effects of isochoric heating, the subsequent evolution of the internal pressure, and associated effects on the local water structure.

From the differential absorption spectra alone, certain conclusions can already be drawn. The absorption increase at the main edge and the decrease in the post-edge region translate in part to a shift of the post-edge absorption to lower energies. Since the post-edge absorption is partially identified with a $\sigma^{*}$ resonance which shifts to lower energies for longer $\mathrm{O} \cdots \mathrm{O}$ distances, i.e. longer hydrogen bonds and vice versa, ${ }^{40,52}$ we conclude that the degree of hydrogen bonding decreases upon vibrational excitation. The induced absorption at energies higher than $545 \mathrm{eV}$ can be attributed to increased disorder of the hydrogen bond network ${ }^{53,54}$. Femtosecond pump- probe experiments in the infrared have shown that water thermalizes within 5 ps upon $\mathrm{OH}$ stretching excitation due to a very rapid cascaded relaxation of vibrational excitations. ${ }^{24}$ Since this thermalization timescale is much faster than the experimental time resolution we attribute the observed absorption change to a temperature increase. Both, the weakening and the increased disorder in hydrogen bonding, are consistent with previous observations from vibrational spectroscopy on water. ${ }^{55,56}$

For comparison with our differential absorption spectra, data from Bergmann et al. ${ }^{50}$ is plotted in Fig. 3B. In the latter experiment, the oxygen $\mathrm{K}$-edge of water was measured via $\mathrm{x}$-ray Raman scattering for sample temperatures of $2{ }^{\circ} \mathrm{C}$ and $22^{\circ} \mathrm{C}$. The differential Raman spectrum in Fig. 3B has been rescaled by a factor of 1.2 and reversed in sign as compared to the original publication to facilitate comparison with the differential absorption spectrum at 200ns time delay.

The overall agreement between the transient absorption data at 200ns and the static XRS data is quite good. However, there are some discrepancies between the two differential spectra such as the energies where $\Delta \mathrm{A}$ changes sign. The lower resolution in the XRS experiment contributes to some extent. Furthermore, the transient absorption data was recorded at higher base sample temperature than the Raman data which suggest that the absorbance change does not depend solely on temperature differences but also on absolute temperature, i.e. the differential Raman spectrum cannot be linearly extrapolated to higher temperatures. Recent higher resolution XRS data on liquid water similarly shows that differential XRS spectra depend on temperature differences and absolute temperature. ${ }^{57}$

We also recorded absorbance changes as a function of pumpprobe delay at specific x-ray probe energies. The results are plotted in Fig. 4. In the left panel, short delay scans are shown that follow the absorbance changes around zero delay for the three indicated $x$-ray energies. The absorbance changes between $75 \mathrm{ps}$ and $200 \mathrm{ps}$ delay are consistent with those measured in the differential spectrum at $150 \mathrm{ps}$ delay (Fig. 3A). These time transients also serve as a cross-check for the baseline $(\Delta \mathrm{A}=0)$ of the differential XAS spectra. In particular, we verified the absence of absorbance changes at $538 \mathrm{eV}$ with an uncertainty below $0.3 \mathrm{mOD}$ by scanning the time delay at this energy (data not shown).

The temporal dynamics in Fig. 4A reflect the 70ps width of the $\mathrm{X}$-ray probe pulses and confirm that the excited water volume reaches a thermalized state well within the time resolution of the experiment. Hence, the data resembles a convolution of the 70ps $\mathrm{x}$-ray pulses and a very fast (step function-like) process. The solid lines are fits of this model to the experimental data with good agreement between model and experiment.

Longer delay scans are displayed in Fig. 4B and 4C. It is evident that the differential signal partially recovers on nanosecond timescales in the pre- and post-edge region while it is essentially constant at the main-edge (consistent with the data in Fig. 3A). We model this recovery with a single exponential $\operatorname{Erf}(\mathrm{t})\left(\mathrm{A}+\mathrm{Be}^{-\mathrm{t} / \tau}\right)-$ and extract a time constant of $\tau=8 \mathrm{~ns} \pm 3 \mathrm{~ns}$ for the data in Fig. 4B. The ratio A/B varies with frequency but is $\sim 2$ in the spectral region of the pre- and post-edge peaks. The fit to the time trace at $537 \mathrm{eV}$ increases with a time constant of $8 \mathrm{~ns}$ $(\mathrm{B}<0)$ but setting $\mathrm{B}=0$ yields equal fit quality. This finding is also consistent with the data in Fig. 3A and shows that within the experimental signal-to-noise ratio no dynamics take place on
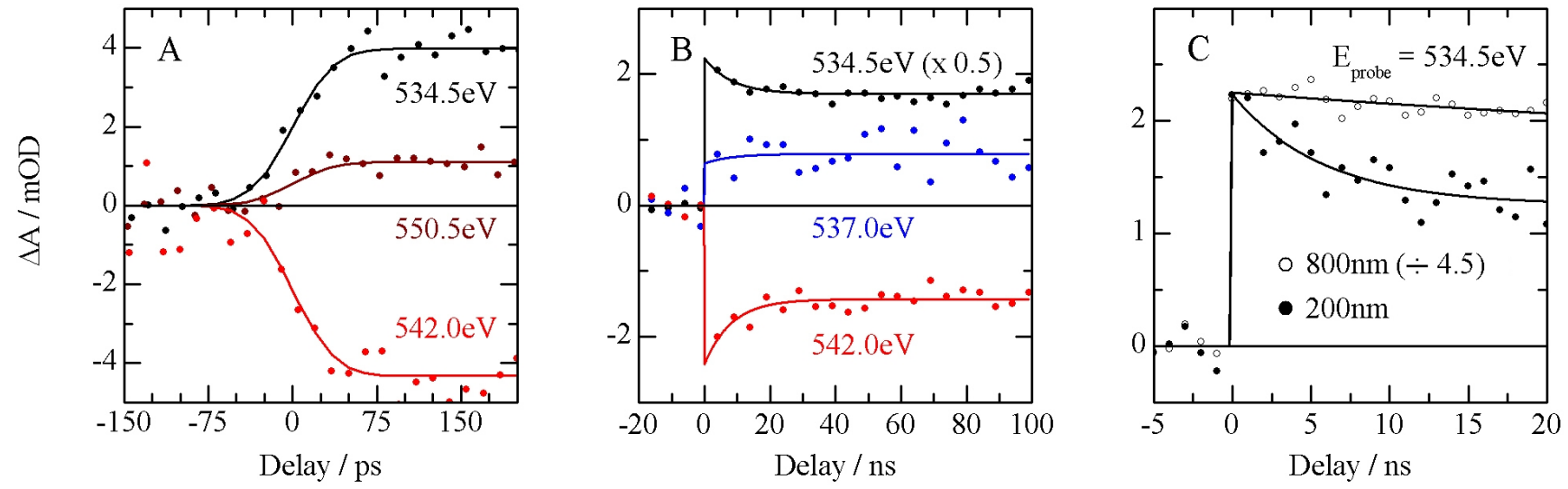

Fig. 4 Sample absorbance change as a function of delay between the $2.95 \mu \mathrm{m}$ excitation pulse and the soft x-ray probe pulse at specific $\mathrm{x}$-ray energies. Solid lines are fits to the data based on an instantaneous absorption change convolved with a 70ps pulse, with A: negligible recovery and B,C: partial single exponential recovery within the measurement window. 
change $\Delta \mathrm{A}$ increases with thickness by 4.5 and the nanosecond timescales in the main edge region.

Importantly, we observe that the time constant for partial signal recovery depends on the sample thickness as is demonstrated in Fig. 4C for delay scans on a thin and thick water film (solid and open circles, respectively). The absorbance corresponding time constants differ by at least the same factor: The thin sample exhibits a time constant of $6 \mathrm{~ns} \pm 2 \mathrm{~ns}$ while the thick sample shows a much slower recovery. Within the accuracy of our 20ns delay scan we extract a time constant of 70ns (with $\mathrm{A} / \mathrm{B}=2$ ). Varying the ratio $\mathrm{A} / \mathrm{B}$ between 1 and 4 varies the time constant by $\pm 40 \mathrm{~ns}$ with similar fit quality.

To evaluate the dependence of the absorbance change $\Delta \mathrm{A}$ on the sample thicknesses we assume that the transmission change $\Delta \mathrm{T}$ is proportional to the absorbed infrared energy, i.e. $\Delta \mathrm{A} \sim$ $\Delta \mathrm{T} / \mathrm{T} \sim\left[1-\exp \left(-\mathrm{x} / \mathrm{l}_{\mathrm{IR}}\right)\right] \exp \left(\mathrm{x} / \mathrm{l}_{\mathrm{xray}}\right)$. Using the absorption lengths $1_{\mathrm{IR}}=800 \mathrm{~nm}$ and $1_{\text {xray }}=1200 \mathrm{~nm}$ at the pre-edge, we derive ratios $\Delta \mathrm{A}_{200 \mathrm{~nm}}: \Delta \mathrm{A}_{400 \mathrm{~nm}}: \Delta \mathrm{A}_{800 \mathrm{~nm}}=1: 2: 4.5$ which match the absorbance changes at $534.5 \mathrm{eV}$ in Fig. $4 \mathrm{~A}-\mathrm{C}$. As a cross-check of the sample thicknesses in Fig. 4C, we extract the experimental transmission ratio of the two thicknesses, $\mathrm{T}_{200 \mathrm{~nm}} /$ $\mathrm{T}_{800 \mathrm{~nm}}=1.75$, and compare it to the theoretical value of $\exp [(800 \mathrm{~nm}-200 \mathrm{~nm}) / 1200 \mathrm{~nm}]=1.65$. The agreement is surprisingly good for this simple model

To explain the changes on nanosecond timescales we note that a transient increase in water temperature at constant volume (isochoric heating) leads to a transient pressure increase that subsequently relaxes via sample expansion. Our sample cell is not a sealed cell but is similar to an open beaker with an initial equilibrium temperature and pressure before the pump pulse excites the sample. Furthermore, the silicon nitride membranes that constitute the transmissive windows of the sample cell are very flexible allowing for sample thicknesses of tens of microns without membrane rupture.

A lower limit for the timescale of the water expansion process can be derived from the (hydrodynamic) speed of sound in water $\left(1.5 \mathrm{~km} \mathrm{~s}^{-1}\right){ }^{58}$ Given the size of the excitation spot $(150 \mu \mathrm{m})$ compared to the nominal sample thickness $(400 \mathrm{~nm})$, transverse expansion can be neglected. Therefore, expansion is predominantly longitudinal and a lower time limit of this process for a thickness of $400 \mathrm{~nm}$ is $270 \mathrm{ps}$. Since water thermalizes within 5ps after $\mathrm{OH}$ stretching excitation the sample is heated at constant volume. The isochoric heating translates into an ultrafast increase in internal pressure that depends on absolute temperature ranging from $0.5 \mathrm{MPa} \mathrm{K}^{-1}$ at $22^{\circ} \mathrm{C}$ to $1.0 \mathrm{MPa} \mathrm{K}^{-1}$ at $50^{\circ} \mathrm{C}$ as calculated from the thermal expansion coefficient and the isothermal compressibility of water. ${ }^{59}$

The maximal temperature change in the water layer can be estimated from the absorbed pump energy, the excited water mass (given by the sample thickness and the pump beam size), and the specific heat capacity of water. When taking into account the Gaussian beam profile and Fresnel reflections at the first sample window, the upper limit for the induced temperature change is $20^{\circ} \mathrm{C}$. However, uncertainties in the exact pump beam size and more importantly, insufficient spatial overlap between the pump and the probe pulse can lead to large errors in temperature estimates. When comparing the relative signal change between the XAS and the XRS data we estimate that the probed temperature change is only $10 \mathrm{~K}$. From this induced temperature change we calculate an initial pressure jump of $8 \mathrm{MPa}$ after $\mathrm{OH}$ stretch excitation.

We infer that the differences in the pre- and post-edge region of the differential absorption spectra in Fig. 3A are associated with an elevated internal pressure at $150 \mathrm{ps}$ delay that has relaxed at $200 \mathrm{~ns}$ through volume expansion on nanosecond timescales. This pressure relaxation is reflected in the time evolution of the transients at the pre- and post-edge in Fig. 4B. Thicker samples have to expand more (on absolute length scales) for the pressure to relax leading to longer relaxation times as is evident in Fig. 4C. This interpretation explains why the differential absorption at 200ns delay (Fig 3A), for which the pressure has relaxed, shows greater similarity with the differential Raman spectrum in Fig. 3B which was recorded at ambient pressure. Raman spectra at the 'oxygen K-edge of water at higher pressures have been measured, ${ }^{60}$ but the high statistical noise in that data precludes a direct comparison with our data.

A time-resolved $x$-ray diffraction study ${ }^{44}$ has found similar increases in temperature and pressure upon ultrafast heating of water. Ultrafast infrared pump-probe experiments in ice $\mathrm{Ih}^{61,62}$ also reported isochoric heating with a pressure increase of $1.3 \mathrm{MPa} \mathrm{K} \mathrm{K}^{-1}$ and subsequent relaxation via adiabatic volume expansion with a time constant of $1 \mathrm{~ns}$. Accounting for the slower speed of sound in water compared to ice $\left(1.5 \mathrm{~km} \mathrm{~s}^{-1} \mathrm{vs}\right.$. $\left.3.9 \mathrm{~km} \mathrm{~s}^{-1}\right)^{58,63}$ we extract an expansion time-constant that is $\sim 6 \mathrm{x}$ larger than expected based on the measurements in ice. A possible explanation is that the silicon nitride windows may act as tamping layers, thereby slowing down the expansion dynamics. ${ }^{64}$ Other experiments monitored water expansion by interferometric surface height changes upon overtone excitation of water vibrations and found water to expand on multiple timescales ranging from nano- to microseconds ${ }^{65}$. We can exclude cooling of the excited sample volume on nanosecond timescales because the thermal diffusivity of neither water nor the window material (silicon nitride) is large enough to allow heat to be dissipated faster than on micro- to millisecond timescales.

Our results show that the main edge region is fairly insensitive to a pressure of $8 \mathrm{MPa}$ whereas the pre- and postedge absorption increases with pressure resulting in similar absorption changes as occur when the temperature is raised at constant pressure. Similar insensitivity of the main edge is also observed in the XRS difference spectrum of $\mathrm{H}_{2} \mathrm{O}$ and $\mathrm{D}_{2} \mathrm{O}$ where the changes in the pre-edge and post-edge follow an expected small difference in temperature. Opposing effects of pressure and temperature on hydrogen bonding have been inferred from pressure-dependent Raman spectroscopy on the $\mathrm{OH}$ stretching vibration of water ${ }^{66}$ but the interplay between temperature and pressure with respect to the oxygen $\mathrm{K}$-edge is not clear and modelling of this edge at elevated pressure is necessary to extract more information from transient $\mathrm{x}$-ray absorption spectra.

\section{Conclusions}

We have performed transient soft x-ray absorption studies of the response of liquid water to an ultrafast excitation of the $\mathrm{OH}$ stretching vibration. We observe thermalization of the water sample within the 70ps time resolution of the experiment that leads to an ultrafast isochoric temperature jump of $10 \mathrm{~K}$ 
accompanied by a pressure increase of $8 \mathrm{MPa}$. Pressure relaxation manifests as a decreased absorption change in the pre- and post-edge region on nanosecond timescales while the main-edge region is fairly insensitive to pressure changes. When comparing our transient XAS spectra to static isobaric XRS data of liquid water at different temperatures we observe discrepancies at sub-nanosecond delays at the pre- and postedge. Absorbance changes partially recover on nanosecond time scales and differential XAS spectra at 200ns delay are in reasonable agreement with XRS data. Both observations suggest that the pre- and post-edge spectral regions of the oxygen $\mathrm{K}$ edge are a sensitive probe of internal pressure. Thus, the effect of pressure on the local structure of liquid water can be studied via transient infrared pump/XAS probe spectroscopy. This work offers a novel approach to study, with high sensitivity, the structural and dynamic effects of pressure and temperature on liquid water - and hydrogen bonding in liquids such as alcohols - and to test theoretical models describing the liquid. Femtosecond $\mathrm{x}$-ray sources will allow new insight into the ultrafast dynamics on (sub-) 100fs timescales providing detailed information on sub-ensembles of water molecules by frequencyselective excitation (vibrational labelling) in the mid- and farinfrared $(\mathrm{THz})$ region of the water spectrum.

\section{Acknowledgements}

This work was supported by the Director, Office of Science, Office of Basic Energy Sciences, the Chemical Sciences, Geosciences, and Biosciences Division under the Department of Energy Contract No. DE-AC02-05CH11231, by the Department of Energy through the Stanford PULSE Institute, and by the National Science Foundation (NSF) CHE-0518637.

\section{Notes and references}

${ }^{a}$ Chemical Sciences Division, Lawrence Berkeley National Laboratory, 1 Cyclotron Road, Berkeley, CA 94720,USA. E-Mail: nhuse@lbl.gov

${ }^{b}$ PULSE Institute, SLAC National Accelerator Laboratory, 2575 Sand Hill Road, Menlo Park, California 94025, USA.

${ }^{c}$ Stanford Synchrotron Radiation Laboratory, P.O.B. 20450, Stanford, CA 94309, USA.

${ }^{d}$ Department of Chemistry, Stanford University, Stanford, California 94305, USA.

${ }^{e}$ Department of Materials Science and Engineering, Stanford University, Stanford, California 94305, USA.

${ }^{f}$ FYSIKUM, Stockholm University, Alba Nova, S-10691 Stockholm, Sweden.

${ }^{g}$ Materials Sciences Division, Lawrence Berkeley National Laboratory, 1 Cyclotron Road, Berkeley, CA 94720, USA.

\$ These authors have contributed equally to this work.

1 D. Eisenberg, W. Kauzmann, The Structure and Properties of Water, Oxford University Press, New York, 1969.

2 B. X. Yang and J. Kirz, Phys. Rev. B, 1987, 36, 1361.

3 E. T. J. Nibbering and T. Elsaesser, Chem. Rev., 2004, 104, 1887.

4 Ph. Wernet, D. Nordlund, U. Bergmann, M. Cavalleri, M. Odelius, H. Ogasawara, L. Å. Näslund, T. K. Hirsch, L. Ojamäe, P. Glatzel, L. G. M. Pettersson, and A. Nilsson, Science 304 (5673), 995 (2004).

5 J. D. Smith, C. D. Cappa, K. R. Wilson, B. M. Messer, R. C. Cohen, and R. J. Saykally, Science, 2004, 306, 851.

6 C. J. Fecko, J. D. Eaves, J. J. Loparo, A. Tokmakoff and P. L. Geissler, Science, 2003, 301, 1698.

7 J. B. Asbury, T. Steinel, C. Stromberg, S. A. Corcelli, C.P. Lawrence, J. L. Skinner and M.D. Fayer, J. Phys. Chem. A, 2004, 108, 1107.

8 M. L. Cowan, B. D. Brunner, N. Huse, J. Dwyer, B. Chugh, E. T. J. Nibbering, T. Elsaesser and R. J. D. Miller, Nature, 2005, 434, 199.
9 A. Paarmann, T. Hayashi, S. Mukamel, and R. J. D. Miller, J. Chem. Phys., 2008, 128, 191103.

10 H. Graener, G. Seifert and A. Laubereau, Phys. Rev. Lett., 1991, 66, 2092.

11 S. Woutersen, U. Emmerichs and H. J. Bakker, Science, 1997, 278, 658

12 G. M. Gale, F. H. G. Gallot, N. Lascoux, S. Bratos and J.-C. Leicknam, Phys. Rev.Lett., 1999, 82, 1068.

13 J. C. Déak, S. T. Rhea, L. K. Iwaki and D. D. Dlott, J. Phys. Chem. A, 2000, 104, 4866 .

14 J. Stenger, D. Madsen, P. Hamm, E. T. J. Nibbering and T. Elsaesser, Phys. Rev. Lett., 2001, 87, 027401.

15 D. Cringus, S. Yeremenko, M. S. Pshenichnikov and D. A. Wiersma, J. Phys. Chem. B, 2004, 108, 10376.

16 T. Steinel, J. Asbury, J. Zheng and M. Fayer, J. Phys. Chem. A, 2004, 108, 10957.

17 S. Woutersen and H. J. Bakker, Nature, 1999, 402, 507.

18 D. Kraemer, M. L. Cowan, A. Paarmann, N. Huse, E. T. J. Nibbering, T. Elsaesser and R. J. D. Miller, Proc. Natl. Acad. Sci., 2008, 105, 437.

19 G. M. Hale and M. R. Querry, Federation Proc., 1973, 12, 555.

20 O. F. A. Larsen and S. Woutersen, J. Chem. Phys., 2004, 121, 12143.

21 N. Huse, S. Ashihara, E. T. J. Nibbering and T. Elsaesser, Chem. Phys. Lett., 2005, 404, 389.

22 Jörg Lindner, Peter Vöhringer, Maxim S. Pshenichnikov, Dan Cringus, Douwe A. Wiersma and Maxim Mostovoy, Chem. Phys. Lett., 2006, 421, 329.

23 S. Ashihara, N. Huse, A. Espagne, E. T. J. Nibbering and T Elsaesser Chem. Phys. Lett., 2006, 424, 66.

24 S. Ashihara, N. Huse, A. Espagne, E. T. J. Nibbering and T Elsaesser $J$. Phys. Chem. A, 2007, 111, 743.

25 P. C. Cross, J. Burnham and P. A. Leighton, J. Am. Chem. Soc., 1937, 59, 1134.

26 E.W. Castner, Jr., Y. J. Chang, Y. C. Chu and G. E.Walrafen, J. Chem. Phys., 1995, 102, 653.

27 C. Rønne, L. Thranea, P.-O. Åstrand, A. Wallqvist, K. V. Mikkelsen and S. R. Keiding, J.Chem. Phys. 107, 5319 (1997).

28 Y. Tominaga, A. Fujiwara, and Y. Amo, Fluid Phase Equilibria, 1997, 144, 323.

29 H. R. Zelsmann, J. Mol. Struct., 1995, 350, 95.

30 K. Winkler, J. Lindner and P. Vohringer, Phys. Chem. Chem. Phys., 2002, 4, 2144.

31 S. Myneni, Y. Luo, L. Å. Näslund, M. Cavalleri, L. Ojamäe, H. Ogasawara, A. Pelmenschikov, P. Wernet, P. Väterlein, C. Heske, Z. Hussain, L. G. M. Pettersson, and A. Nilsson, J. Phys.: Cond. Mat., 2002, 14, L213.

32 J. H. Guo, Y. Luo, A. Augustsson, J. E. Rubensson, C. Såthe, H. Ågren, H. Siegbahn, and J. Nordgren, Phys. Rev. Lett, 2002, 89, 137402.

33 L.-Å. Näslund, J. Lüning, Y. Ufuktepe, H. Ogasawara, Ph. Wernet, U. Bergmann, L. G. M. Pettersson, and A. Nilsson, J. Phys. Chem. B, 2005, 109, 13835.

34 D. Nordlund, H. Ogasawara, H. Bluhm, O. Takahashi, M. Odelius, M. Nagasono, L. G. M. Pettersson, and A. Nilsson, Phys. Rev. Lett., 2007, 99, 217406 .

35 T. Tokushima, Y. Harada, O. Takahashi, Y. Senba, H. Ohashi, L. G. M. Pettersson, A. Nilsson, and S. Shin, Chem. Phys. Lett., 2008, 460, 387.

36 M. V. Fernandez-Serra, and E. Artacho, Phys. Rev. Lett., 2006, 96, 016404.

37 B. Hetenyi, F. De Angelis, P. Giannozzi, and R. Car, J. Chem. Phys., 2004, 120, 8632.

38 M. Cavalleri, M. Odelius, D. Nordlund, A. Nilsson, and L. G. M. Pettersson, Phys. Chem. Chem. Phys., 2005, 7, 2854.

39 D. Prendergast, and G. Galli, Phys. Rev. Lett., 2006, 96, 215502.

40 M. Odelius, M. Cavalleri, A. Nilsson, and L. G. M. Pettersson, Phys. Rev. B, 2006, 73, 024205.

41 J. D. Smith, C. D. Cappa, B. M. Messer, W. S. Drisdell, R. C. Cohen, and R. J. Saykally, J. Phys. Chem. B, 2006, 110, 20038.

42 J. S. Tse, D. M. Shaw, D. D. Klug, S. Patchovskii, G. Vanko, G. Monaco and M. Krisch, Phys. Rev. Lett., 2008, 100, 095502.

43 M. Iannuzzi, J. Chem. Phys., 2008, 128, 204506.

44 A.M. Lindenberg, Y. Acremann, D. P. Lowney, P. A. Heimann, T. K. Allison, T. Matthews and R. W. Falcone, J. Chem. Phys., 2005, 122, 204507. 
45 M . Wulff, S. Bratos, A. Plech, R. Vuilleumier, F. Mirloup, M. Lorenc, Q. Kong and H. Ihee, J. Chem. Phys., 2006, 124, 034501.

46 P. Georgiou, J. Vincent, M. Andersson, A. B. Wohri, P. Gourdon, J. Poulsen, J. Davidsson and Richard Neutze, J. Chem. Phys., 2006, 124, 234507.

47 N. Huse, T. K. Kim, M. Khalil, L. Jamula, J. K. McCusker and R. W. Shoenlein, Ultrafast Phenomena XVI, Springer-Verlag, Berlin Heidelberg, 2009, in press.

48 P.A. Heimann, H.A. Padmore and R.W. Schoenlein, AIP Conference Proceedings, 2004, 705, 1407.

49 P. A. Heimann, T. E. Glover, D. Plate, H. J. Lee, V. C. Brown, H. A. Padmore, and R. W. Schoenlein, AIP Conference Proceedings, 2007, 879, 1195.

50 U. Bergmann, D. Nordlund, Ph. Wernet, M. Odelius, L. G. M. Pettersson and A. Nilsson, Phys. Rev. B, 2007, 76, 024202.

$51 \mathrm{Ph}$. Wernet, G. Gavrila, K. Godehusen, C.Weniger, E.T.J. Nibbering, T. Elsaesser, and W. Eberhardt, Apl. Phys. A, 2008, 92, 511.

52 J. Stöhr, NEXAFS Spectroscopy, Springer, Berlin, 1992.

$53 \mathrm{Ph}$. Parent, C. Laffon, C. Mangeney, F. Bournel, M. Tronc, J. Chem. Phys., 2002, 117, 10842.

$54 \mathrm{Ph}$. Wernet, D. Testemale, J.-L. Hazemann, R. Argoud, P. Glatzel, L. G. M. Pettersson, A. Nilsson, and U. Bergmann, J. Chem. Phys., 2005, 123, 154503.

55 G. E. Walrafen, J. Phys. Chem., 1967, 41, 114.

56 G. E. Walrafen, M.S. Hokmabadi and W.-H. Yang, J. Phys. Chem., 1988, 92, 2433

57 D. Nordlund, unpublished.

58 W. D. Wilson, J. Acoust. Soc. Am., 1959, 31, 1067.

59 R. A. Fine and F. J. Millero, J. Chem. Phys., 1973, 59, 5529.

60 H. Fukui, S. Huotari, D. Andraul, T. Kawamoto, J. Chem. Phys. 127 (2007) 134502.

61 H. Iglev, M. Schmeisser, K. Simeonidis, A. Thaller and A. Laubereau, Nature, 2006, 439, 183.

62 M. Schmeisser, A. Thaller, H. Iglev and A. Laubereau, New J. Phys., 2006, 8, 104.

63 Gagnon R E, Kiefte H, Clouter M J and Whalley E, J. Chem. Phys., 1988, 89, 4522.

64 S.L. Johnson, P. A. Heimann, A. G. MacPhee, A. M. Lindenberg, O. R. Monteiro, Z. Chang, R. W. Lee, and R. W. Falcone, Phys. Rev. Lett., 2005, 94, 057407.

65 J. Hobley, Y. Kuge, S. Gorelik, M. Kasuya, K. Hatanaka, S. Kajimoto, and Hiroshi Fukumura, Phys. Chem. Chem. Phys, 2008, 10, 5256.

66 T. Kawamoto, S. Ochiai and H. Kagi, J. Chem. Phys., 2004, 120, 5867. 